\title{
Management of hip fracture in the older people: rationale and design of the Italian consensus on the orthogeriatric co-management
}

\author{
Antonio De Vincentis ${ }^{1}$. Astrid Ursula Behr ${ }^{2}$. Giuseppe Bellelli, ${ }^{3,4}$ Marco Bravi ${ }^{5}$. Anna Castaldo ${ }^{6} \cdot$ Claudio Cricelli $^{7}$. \\ Lucia Galluzzo $^{8}$. Giovanni lolascon ${ }^{9} \cdot$ Stefania Maggi $^{10} \cdot$ Emilio Martini $^{11} \cdot$ Alberto Momoli $^{12} \cdot$ Graziano Onder $^{8}$. \\ Marco Paoletta ${ }^{9} \cdot$ Mauro Roselli $^{13} \cdot$ Mauro Ruggeri $^{14} \cdot$ Fabio Santacaterina $^{5} \cdot$ Luigi Tritapepe $^{15} \cdot$ Amedeo Zurlo $^{16}$. \\ Raffaele Antonelli Incalzi ${ }^{1}$
}

Received: 10 March 2020 / Accepted: 17 April 2020 / Published online: 1 May 2020

(c) Springer Nature Switzerland AG 2020

\begin{abstract}
Background Hip fracture (HF) is a burdening health problem in older people. The orthogeriatric approach has been shown to favour functional recovery and reduce mortality, but its implementation in clinical practice cannot rely upon shared management protocols and greatly varies among different healthcare systems. Here, we present the rationale and design of the Italian consensus document on the management of HF in older people.

Methods A panel of multidisciplinary experts from ten Italian scientific societies involved in the care of HF and including geriatricians, orthopaedics, anaesthesiologists, physiatrists and general practitioners, will join to establish the content validity of a list of statements. A Delphi consensus methodology will be applied to obtain the opinions of the panel and to provide the final recommendations.

Objectives The document will include indications on the following relevant topics: (1) optimal care path of older subjects with HF; (2) management of comorbidities and pre-operative alteration of physiological parameters; (3) management of selected categories of patients at expected increased risk of adverse outcomes; (4) continuity of care out of hospital; (5) screening and correction of risk factors for HF in older subjects; (6) information and divulgation of shared management strategies. The objective of the consensus will be to inform clinicians, patients, researchers, and health policy makers about the best management strategies for HF in older people and their inherent limitations, thus facilitating communication between stakeholders and promoting the most cost/effective models of care.
\end{abstract}

Keywords Hip fracture $\cdot$ Elderly $\cdot$ Orthogeriatric co-management

\section{State of the art}

Hip fracture (HF) is a major health care problem in older subjects. Its incidence has steadily increased in the last decades in line with the progressive population ageing [1]. In Italy around 90-100,000 HF are registered every year [2], with subjects aged 85 years or more accounting for the $40-45 \%$ of total hospital admissions for this problem, despite they represent only the $2.5 \%$ of the Italian population [1]. In general, morbidity and mortality are a considerable issue. Indeed, approximately the $5-10 \%$ of patients die in a month and the $20-25 \%$ in a year [2]. Moreover, only

Antonio De Vincentis

a.devincentis@unicampus.it

Extended author information available on the last page of the article one-third of subjects surviving HF will conduct an independent life, while around a half will develop a permanent disability [3-5]. Healthcare costs are comparably high and have already outweighed those for acute myocardial infarctions [6], thus confirming the very high economic burden of these fractures.

In this scenario, many factors are known to play a role in influencing the occurrence of adverse health outcomes. Performing surgery on the day of, or the day after, hospital admission (within $48 \mathrm{~h}$ ) has been demonstrated to significantly shorten the length of hospital stay, favouring the return to independent life, and to reduce the incidence of pressure ulcers and, ultimately, of mortality $[7,8]$. The optimal management of HF goes far beyond the correct orthopaedic surgical management, and many other coexisting patients' conditions deserve accurate consideration. 
Indeed, patients with pre-fracture cognitive or neurological impairment are more likely to develop delirium [9], as well as those with severe comorbidity or pre-existing disability are more likely to experience worse outcomes after HF [5, 10]. Furthermore, the occurrence of post-operative complications, such as chest infections or congestive heart failure, have a major impact on mortality [11].

To cope with these problems, the cooperation between geriatricians and orthopaedic surgeons has been regarded as the key solution since the 1960s [12], and different orthogeriatric management models have been identified (Table 1). Each has its own peculiarities and altogether have been evaluated in many different studies compared to traditional care, defined as the absence of geriatric consultation or consultation requested by the orthopaedic surgeon only after a clinical problem has presented (reactive or usual model) [13]. Briefly, the rehabilitative model involves geriatricians only in the post-operative and rehabilitative phase, while all the others involve them earlier after HF, and the amount of geriatric commitment is variable from planned geriatric consultations in orthopaedic ward (consultative model), to a more integrated approach based on a shared patient management by both orthopaedics and geriatricians (integrated model). Overall, two recent meta-analysis highlighted orthogeriatric co-management as the best approach to decrease short- and long-term mortality and reduce the length of hospital stay [14]. This approach is also able to improve functional recovery and independence in the daily activities at follow-up, compared to traditional care [15]. However, available studies have failed to provide sufficient evidence for the prominence of a specific orthogeriatric management model among consultative, rehabilitative or integrated [16]. In addition, the application of orthogeriatric principles in clinical practice greatly varies depending upon the healthcare system organization. For instance, in the United Kingdom the orthogeriatric approach has been widely applied since it was prioritized by the introduction of higher financial incentives for compliant hospitals, and this initiative was shown to be mirrored by improved outcomes [17]. Several countries have developed their own guidelines or consensus reports, based on consolidated experience and the cultural attitude of the country $[18,19]$. Conversely, in many other countries, including Italy, the application of the orthogeriatric model is much more heterogeneous with great differences among regions. Shared nation-level management protocols are also lacking.

Hereby, we present the design of the Italian consensus document for the management of HF in older people. Following the spirit of a concrete cultural and scientific integration, this document will be implemented and endorsed by ten Italian scientific organizations Società Italiana Geriatria e Gerontologia (SIGG), Associazione Italiana di Psicogeriatria (AIP), Società Italiana di Geriatria Ospedale e Territorio (SIGOT), Società Italiana di Medicina Generale (SIMG), Società Italiana di Anestesia Analgesia Rianimazione e Terapia Intensiva (SIAARTI), Società Italiana di Ortopedia e Traumatologia (SIOT), Società Italiana di Medicina Fisica e Riabilitativa (SIMFER), Società Italiana di Farmacologia (SIF), Consiglio Nazionale delle Ricerche (CNR), Istituto Superiore Sanità (ISS), and will collect practical statements on selected relevant issues in the management of HF in older people. The document will be based on an adaptation of the current guidelines (mainly the NICE Guidelines on the management of hip fracture in adults [18]) and in accordance with the Italian situation and the expert opinion of the multidisciplinary panel.

\section{Design of the consensus}

The consensus will be focused on the following relevant topics, representing still open fields of discussion in the management of geriatric HF (Fig. 1).

\section{Optimal care path of older subjects with HF}

The optimal care of patients with HF along with the specific orthogeriatric organization to be more conveniently applied is still a matter of debate. The integrated care model seems to emerge above the others for lower mortality, length of hospital stay and better functional outcomes [16], but definite conclusions cannot be drawn in absence of large multicentric randomized trials with head-to-head comparison of different models. In any case, it's undoubtful that the quality of care

Table 1 Types of orthogeriatric models of care of older subjects with hip fracture

\begin{tabular}{lllll}
\hline Model & Reactive or usual & Consultative & Rehabilitative & Integrated care \\
\hline Admission & Variable & Variable & After surgery & Before surgery \\
Care manager & Orthopaedics & Orthopaedics & Geriatrician/Physiatrist & Geriatrician \\
Geriatric assistance & When required by orthopaedics & Daily & Daily or with variable schedule & Daily \\
Multidisciplinary care & Based on occurring clinical & Suggested by geriatrician & Coordinated by Geriatrician/ & Shared coordination by \\
& problems & & Physiatrist & Geriatrician/Ortho- \\
& & & & paedic \\
\hline
\end{tabular}




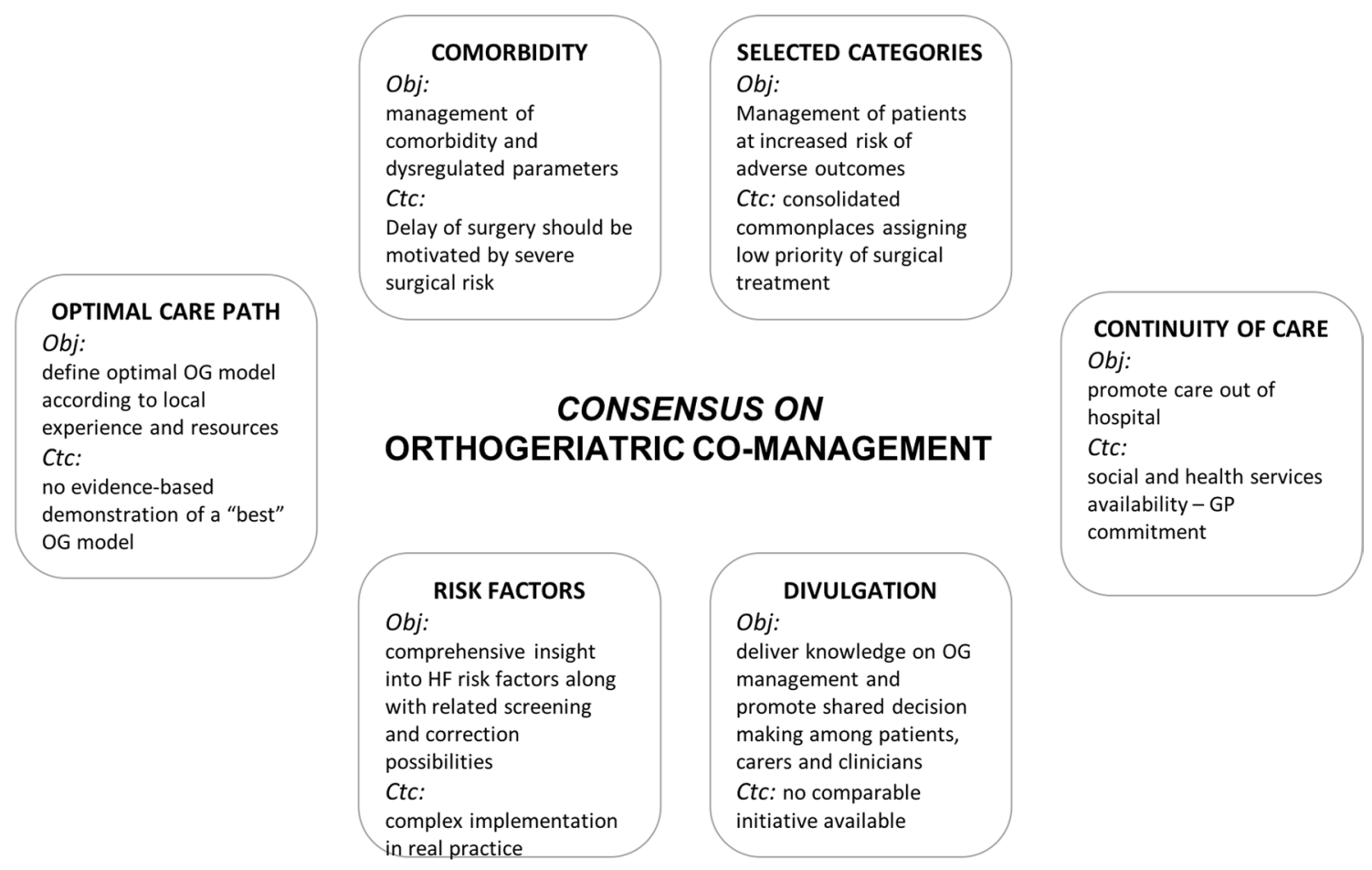

Fig. 1 The orthogeriatric co-management model: objectives and criticisms related to the main topics of interest. $O b j$ objective, $C t c$ criticism, $O G$ orthogeriatric

is influenced by the configuration of orthopaedic and geriatric medicine services, hospital protocols and processes, and the degree to which a multidisciplinary approach to care is taken $[14,15]$. Hence, each hospital should implement a dedicated model for geriatric HF, based on local experience, economic availability and healthcare resources, taking care of collecting detailed data on patients, processes of care, and outcomes to be able to participate in audit processes.

\section{Management of comorbidities and pre-operative alteration of physiological parameters}

Since the great majority of subjects with HF is older than 70 years and presents with multiple coexisting medical problems, a related -often overlooked-issue is the timely management of patients' clinical conditions. Approximately $50-60 \%$ of patients have got at least one major comorbidity (cardio-cerebrovascular, dementia or chronic pulmonary diseases in around the $30-5 \%, 20 \%$ and $10-15 \%$ of cases, respectively) and half of them is affected by three or more co-occurring diseases $[11,20]$. In these patients, the excess mortality risk within 1 year from HF is higher than could be explained by HF per se [20]. Noteworthy, this increased mortality risk is also the result of coexisting dysregulation of other parameters, such as haemoglobin, creatinine, and electrolytes. Indeed, pre-operative anaemia is a frequent problem ( $\sim 50 \%$ of subjects) and can significantly worsen prior to surgery due to bleeding (particularly in extracapsular HF), generally leading to increased blood transfusion requirements [21]. Similarly, many patients with HF are affected by chronic kidney failure ( $\sim 2 \%$ of subjects) and a quarter of them presents acute kidney failure at hospital admission [22]. Moreover, electrolyte disturbances are very prevalent in this population. In particular, hyperkalaemia has been associated with increased mortality, even independently of potential confounders [23]. Altogether, even though organizational strategies are generally given much more importance, the correct clinical management of coexisting disturbances play a pivotal role in influencing outcomes. As such, much attention should be paid to their early assessment and correction, whenever possible.

\section{Management of selected categories of patients at expected increased risk of adverse outcomes}

Hitherto, no evidence-based indications are available to help clinicians in the complex pre-operative evaluation of patients with HF, so that, besides often underestimated clinical features (e.g., kidney failure, anaemia, electrolyte disturbances), other conditions are usually considered too much due to consolidated commonplaces. For instance, patients with cognitive decline or neurological disorders are generally given low priority for surgery and rehabilitation after HF, since they are thought to have lower chances of functional recovery. A 
similar approach is used in case of a relapsed HF. Actually, different studies evidenced that ability to walk and independence in activities of daily living before HF are the leading prognostic factors for motor recovery, rather than cognitive or neurological impairment themselves [24, 25]. Similarly, length of hospital stay and functional outcomes after a second HF do not differ from those observed after the first HF [26]. There is also growing evidence that frailty status can have a prognostic role in HF patients, being associated with the major short- and long-term adverse outcomes, such as postoperative complications, hospital length of stay, lower functional recovery, hospital readmission and mortality [27, 28].

\section{Continuity of care out of hospital}

It should be emphasized that the care of subjects with HF does not end with hospital discharge. The continuity of care out of hospital is of great importance for maintaining and improving the achieved functional level and preventing deconditioning and isolation. Early discharge directly at home is the preferable choice in the patient's perspective when stable post-operative clinical conditions and early mobilization in the acute ward are achievable, and when optimal care and the logistic situation at home is available. To this purposes, the commitment of general practitioner and local health and social authorities is welcome. However, studies have also shown that the extent of functional recovery after HF is also dependent on the choice of post-hospital setting of care and on the intensity and duration of provided rehabilitation [29]. Rehabilitation can also be effective for patients with moderate to severe dementia [30]. Dedicated plans should be designed to cope with patients' necessity and provide the adequate level of care to prevent recurrent falls and relapse HF.

\section{Screening and correction of risk factors for HF in older subjects}

HF in older subjects is almost invariably secondary to osteoporosis and falls. While strategies to screen and treat osteoporosis are well-known, their implementation in older people is not optimal. Moreover, much lower is the awareness on behaviours that could favour or contrast HF, or on available tools to screen for the risk of fall and prevent their occurrence. Calcium and vitamin D supplementation are usually regarded as the milestones of nutrition for bone fragility, but sodium and potassium are known to modulate urinary calcium excretion, and vitamin A, C and $\mathrm{K}$ play important roles in bone remodelling [31-33]. Furthermore, moderation of animal food consumption and an increased ratio of vegetable/animal protein intake may confer a protective effect on $\mathrm{HF}$, due to the neutralizing effect of vegetable protein on endogenous acid production, which is a critical determinant of bone fragility [34]. An enlarged perspective would include the consideration of a broad spectrum of risk factors, including those for falls and related to patients' life environment (e.g., low lightning, slippery conditions, absence of assistive device in bathrooms), gait and balance disturbances, impaired vision, urinary incontinence, and selected comorbidities and medications inducing sedation or orthostatic hypotension [35].

An integrated approach to the screening and the care of all these factor would be suitable to efficiently impact on $\mathrm{HF}$ incidence in the older population.

\section{Information and divulgation of shared management strategies}

As previously highlighted, the optimal care of patients with HF is based on the involvement of a number of different physicians along with the commitment of the caregivers. Health policy maker plays a relevant role in allocating resources and organizing services to make all this complex network working properly. Beside defining the optimal theoretical management based on updated evidence, divulgation of shared strategies facilitates communication between stakeholders and avoids unwarranted variation in care.

To face with all these topics, a panel of multidisciplinary experts in geriatric medicine, orthopaedics, anaesthesiology, physiatry and general medicine will be invited to join the consensus work and to establish the content validity of a list of statements answering questions on intervention review. Review questions will be defined using the PICO framework (patient, intervention, comparison and outcome). A Delphi consensus methodology will be applied to obtain the opinions of the expert panel of specialists. The panel will be divided into groups, covering the three main areas identified: emergency department and preoperative phase, intervention, and postoperative phase. In the preliminary Delphi round, each group will be assigned a specific subgroup of questions for each area, and will draft preliminary recommendations in response to each question, supported by appropriate references. In addition, a preliminary assessment of the overall quality of the evidence for each question will be made at this time. In subsequent Delphi rounds, the whole panel will have the opportunity to suggest modifications to any of the questions and recommendations. The panel will adopt the United States Preventative Services Task Force (USPFTF) system for grading the quality of evidence and strength of recommendations [36]. A final consensus conference will be held, in which the panel will finalize the wording, quality of evidence and strength of each recommendation. 


\section{Expected achievements and conclusions}

A long time has elapsed since Devas and Irvine firstly described the role of the geriatrician in the management of older orthopaedic patients with HF in 1963 [12]. Although the HF incidence substantially increased in the following decades in line with the progressive population aging and increasing comorbidity, recent data show that mortality significantly decreased from 1980 until now, testifying to the effectiveness of the orthogeriatric approach [37]. Notwithstanding, these data comes from countries that more efficiently applied the multidisciplinary care of patients with $\mathrm{HF}$, and the real situation is much more variegated. In Italy, the orthogeriatric organization depends upon local resources, experience and sensibility, and the usual reactive approach is still widely observed. This translates in just more than $60 \%$ of patients receiving surgery within $48 \mathrm{~h}$ from hospital admission, compared to an expected standard of $80 \%$, with a considerable variability between different regions [2]. A multicentre study, on more than 3000 patients recruited from 14 Italian hospitals, clearly demonstrated the huge variability among centres with regard not only to the time from fracture to surgery but also to other six key-performance indicators [38]. The heterogeneous picture is likely to be favoured by the absence of shared clinical care standards that could help clinicians and policy makers to provide an adequate level of care for patients with HF.

The consensus work presented hereby is expected (1) to define the appropriate care of older patients with HF, providing indications for a multidisciplinary management, according to local experience and resources; (2) to provide practical indications on management of clinical problems (such as preoperative anemia, kidney failure, cognitive function, electrolyte disturbances), which are frequently deserved less attention compared to organizational topics; (3) to clarify, confuting consolidated commonplaces, the most appropriate management of selected categories of patients at expected increased risk of adverse outcomes (such as those cognitively impaired, with relapsed HF or with neurological problems); (4) to ensure continuity of care out of hospital and strict liaison with the GP throughout the whole therapeutic path; (5) to discuss the main risk factors for HF in the practical perspective of addressing both screening and correction, if possible, of them; (6) to inform clinicians, patients, researchers, and health policy makers about how to tailor the management strategy to the individual patient.

\section{Funding None.}

\section{Compliance with ethical standards}

Conflict of interest Nothing to disclose.
Statement of human and animal rights This paper is an Editorial and no humans or animals were involved.

Informed consent For this type of study formal consent is not required.

\section{References}

1. Piscitelli P, Feola M, Rao C et al (2014) Ten years of hip fractures in Italy: for the first time a decreasing trend in elderly women. World J Orthop 5:386-391. https://doi.org/10.5312/wjo.v5.i3.386

2. Programma Nazionale Esiti-PNE 2018-Home. https://pne. agenas.it/index.php?lang=IT. Accessed 3 Mar 2020

3. Haentjens P, Magaziner J, Colón-Emeric CS et al (2010) Meta-analysis: excess mortality after hip fracture among older women and men. Ann Intern Med 152:380-390. https://doi. org/10.7326/0003-4819-152-6-201003160-00008

4. Boonen S, Autier P, Barette M et al (2004) Functional outcome and quality of life following hip fracture in elderly women: a prospective controlled study. Osteoporos Int 15:87-94. https://doi. org/10.1007/s00198-003-1515-z

5. Maggi S, Siviero P, Wetle T et al (2010) A multicenter survey on profile of care for hip fracture: predictors of mortality and disability. Osteoporos Int 21:223-231. https://doi.org/10.1007/s0019 8-009-0936-8

6. Piscitelli P, Iolascon G, Gimigliano F et al (2007) Incidence and costs of hip fractures compared to acute myocardial infarction in the Italian population: a 4-year survey. Osteoporos Int 18:211219. https://doi.org/10.1007/s00198-006-0224-9

7. Lee DJ, Elfar JC (2014) Timing of hip fracture surgery in the elderly. Geriatr Orthop Surg Rehabil 5:138-140. https://doi. org/10.1177/2151458514537273

8. Pincus D, Ravi B, Wasserstein D et al (2017) Association between wait time and 30-day mortality in adults undergoing hip fracture surgery. JAMA 318:1994-2003. https://doi.org/10.1001/ jama.2017.17606

9. Bellelli G, Carnevali L, Corsi M et al (2018) The impact of psychomotor subtypes and duration of delirium on 6-month mortality in hip-fractured elderly patients. Int J Geriatr Psychiatry. https:// doi.org/10.1002/gps.4914

10. Uriz-Otano F, Uriz-Otano JI, Malafarina V (2015) Factors associated with short-term functional recovery in elderly people with a hip fracture. Influence of cognitive impairment. J Am Med Dir Assoc 16:215-220. https://doi.org/10.1016/j.jamda.2014.09.009

11. Roche JJW, Wenn RT, Sahota O et al. (2005) Effect of comorbidities and postoperative complications on mortality after hip fracture in elderly people: prospective observational cohort study. BMJ 331:1374. https://doi.org/10.1136/bmj.38643.663843.55

12. Devas MBIB (1963) The geriatric orthopaedic unit. J Bone Jt Surg Br 49:186-187

13. Sabharwal S, Wilson H (2015) Orthogeriatrics in the management of frail older patients with a fragility fracture. Osteoporos Int 26:2387-2399. https://doi.org/10.1007/s00198-015-3166-2

14. Grigoryan KV, Javedan H, Rudolph JL (2014) Orthogeriatric care models and outcomes in hip fracture patients: a systematic review and meta-analysis. J Orthop Trauma 28:e49-55. https:// doi.org/10.1097/BOT.0b013e3182a5a045

15. Mukherjee K, Brooks SE, Barraco RD et al (2020) Elderly adults with isolated hip fractures-orthogeriatric care versus standard care: a practice management guideline from the Eastern Association for the surgery of Trauma. J Trauma Acute Care Surg 88:266-278. https://doi.org/10.1097/TA.0000000000002482

16. Kammerlander C, Roth T, Friedman SM et al (2010) Ortho-geriatric service-a literature review comparing different models. 
Osteoporos Int 21:S637-646. https://doi.org/10.1007/s0019 8-010-1396-x

17. Oakley B, Nightingale J, Moran CG et al. (2017) Does achieving the best practice tariff improve outcomes in hip fracture patients? An observational cohort study. BMJ Open 7:e014190. https://doi. org/10.1136/bmjopen-2016-014190

18. Overview I Hip fracture: management I Guidance I NICE. https:// www.nice.org.uk/guidance/cg124. Accessed 3 Mar 2020

19. https://anzhfr.org/guidelines-and-standards/. Accessed $28 \mathrm{Apr}$ 2020

20. Lunde A, Tell GS, Pedersen AB et al (2019) The role of comorbidity in mortality after hip fracture: a nationwide norwegian study of 38,126 women with hip fracture matched to a general-population comparison cohort. Am J Epidemiol 188:398-407. https://doi. org/10.1093/aje/kwy251

21. Puckeridge G, Terblanche M, Wallis M et al. (2019) Blood management in hip fractures; are we leaving it too late? A retrospective observational study. BMC Geriatrics 19:79. https://doi. org/10.1186/s12877-019-1099-x

22. Porter CJ, Moppett IK, Juurlink I et al (2017) Acute and chronic kidney disease in elderly patients with hip fracture: prevalence, risk factors and outcome with development and validation of a risk prediction model for acute kidney injury. BMC Nephrology 18:20. https://doi.org/10.1186/s12882-017-0437-5

23. Norring-Agerskov D, Madsen CM, Abrahamsen B et al (2017) Hyperkalemia is associated with increased 30-day mortality in hip fracture patients. Calcif Tissue Int 101:9-16. https://doi. org/10.1007/s00223-017-0252-9

24. Di Monaco M, Vallero F, Di Monaco R et al (2003) Functional recovery and length of stay after hip fracture in patients with neurologic impairment. Am J Phys Med Rehabil 82:143-148. https ://doi.org/10.1097/00002060-200302000-00010 (quiz 149-151, 157)

25. Beloosesky Y, Grinblat J, Epelboym B et al (2002) Functional gain of hip fracture patients in different cognitive and functional groups. Clin Rehabil 16:321-328. https://doi.org/10.1191/02692 $15502 \mathrm{cr} 497 \mathrm{oa}$

26. Monaco MD, Monaco RD, Manca M et al. (2002) Functional recovery and length of stay after recurrent hip fracture. Am J Phys Med Rehabil 81:86-89

27. Chen C-L, Chen C-M, Wang C-Y et al (2019) Frailty is associated with an increased risk of major adverse outcomes in elderly patients following surgical treatment of hip fracture. Scientific Reports 9:1-9. https://doi.org/10.1038/s41598-019-55459-2

28. Inoue T, Misu S, Tanaka T et al (2019) Frailty defined by 19 items as a predictor of short-term functional recovery in patients with hip fracture. Injury 50:2272-2276. https://doi.org/10.1016/j.injur y.2019.10.011

29. Seitz DP, Gill SS, Austin PC et al (2016) Rehabilitation of older adults with dementia after hip fracture. J Am Geriatr Soc 64:4754. https://doi.org/10.1111/jgs.13881

30. Morghen S, Gentile S, Ricci E et al (2011) Rehabilitation of older adults with hip fracture: cognitive function and walking abilities. J Am Geriatr Soc 59:1497-1502. https://doi.org/10.111 1/j.1532-5415.2011.03496.x

31. Hao G, Zhang B, Gu M et al (2017) Vitamin K intake and the risk of fractures. Medicine (Baltimore). https://doi.org/10.1097/ MD.0000000000006725

32. Feskanich D, Singh V, Willett WC et al. (2002) Vitamin A intake and hip fractures among postmenopausal women. JAMA 287:4754. https://doi.org/10.1001/jama.287.1.47

33. Malmir H, Shab-Bidar S, Djafarian K (2018) Vitamin C intake in relation to bone mineral density and risk of hip fracture and osteoporosis: a systematic review and meta-analysis of observational studies. Br J Nutr 119:847-858. https://doi.org/10.1017/ S0007114518000430

34. Frassetto LA, Todd KM, Morris RC et al. (2000) Worldwide incidence of hip fracture in elderly women: relation to consumption of animal and vegetable foods. J Gerontol A Biol Sci Med Sci 55:M585-592. https://doi.org/10.1093/gerona/55.10.m585

35. Cosman F, de Beur SJ, LeBoff MS et al (2014) Clinician's guide to prevention and treatment of osteoporosis. Osteoporos Int 25:2359-2381. https://doi.org/10.1007/s00198-014-2794-2

36. Grade Definitions-US Preventive Services Task Force. https:// www.uspreventiveservicestaskforce.org/Page/Name/grade-defin itions. Accessed 4 Mar 2020

37. Pedersen AB, Ehrenstein V, Szépligeti SK et al (2017) Thirtyfive-year trends in first-time hospitalization for hip fracture, 1-year mortality, and the prognostic impact of comorbidity: a Danish Nationwide Cohort Study, 1980-2014. Epidemiology 28:898-905. https://doi.org/10.1097/EDE.0000000000000729

38. Ferrara MC, Andreano A, Tassistro E et al (2020) Three-year national report from the Gruppo Italiano di Ortogeriatria (GIOG) in the management of hip-fractured patients. Aging Clin Exp Res. https://doi.org/10.1007/s40520-020-01488-1

Publisher's Note Springer Nature remains neutral with regard to jurisdictional claims in published maps and institutional affiliations.

\section{Affiliations}

\section{Antonio De Vincentis ${ }^{1}$ - Astrid Ursula Behr ${ }^{2}$ - Giuseppe Bellelli ${ }^{3,4} \cdot$ Marco Bravi $^{5}$ - Anna Castaldo ${ }^{6}$. Claudio Cricelli ${ }^{7}$. Lucia Galluzzo $^{8}$. Giovanni lolascon ${ }^{9}$. Stefania Maggi ${ }^{10}$. Emilio Martini ${ }^{11}$. Alberto Momoli ${ }^{12}$. Graziano Onder ${ }^{8}$. Marco Paoletta ${ }^{9} \cdot$ Mauro Roselli $^{13} \cdot$ Mauro Ruggeri $^{14} \cdot$ Fabio Santacaterina $^{5} \cdot$ Luigi Tritapepe $^{15} \cdot$ Amedeo Zurlo $^{16}$. Raffaele Antonelli Incalzi ${ }^{1}$}

1 Geriatric and Gerontology Department, Campus BioMedico University of Rome, via Alvaro del Portillo, 200, 00128 Rome, Italy

2 Operative Unit of Anesthesia and Resuscitation, Hospital of Camposampiero, Padua, Italy

3 Acute Geriatric and Orthogeriatric Unit, S. Gerardo Hospital, Monza, Italy
4 Milano-Bicocca University, Milan, Italy

5 Physical and Rehabilitation Medicine Department, Campus Bio-Medico University of Rome, Rome, Italy

6 IRCCS SMN Fondazione Don Gnocchi, Milano, Italy

7 Italian College of General Practitioners, Florence, Italy 
8 Department of Cardiovascular, Endocrine-Metabolic Diseases and Ageing, Istituto Superiore di Sanità, Rome, Italy

9 Department of Medical and Surgical Specialties and Dentistry, University of Campania "Luigi Vanvitelli", Naples, Italy

10 Neuroscience Institute, Aging Branch, CNR, Padua, Italy

11 Orthogeriatric Unit, Azienda Ospedaliero Universitaria di Modena, Ospedale Civile Di Baggiovara, Modena, Italy

12 Orthopedic and Traumatology Unit, San Bortolo Hospital, Vicenza, Italy
13 Orthopedic and Traumatology Unit, "Maria Vittoria" Hospital, Torino, Italy

14 Società Italiana Di Medicina Generale (SIMG), Firenze, Italy

15 Operative Unit of Anesthesia and Intensive Care Medicine, San Camillo-Forlanini Hospital, Rome, Italy

16 Geriatric and Orthogeriatric Unit, Arcispedale S. Anna, University of Ferrara, Ferrara, Italy 